\title{
Towards a Research Agenda for Social Work Practice in Virtual Worlds
}

\author{
Scott Anstadt \\ Ashley Burnette \\ Shannon Bradley
}

\begin{abstract}
Due to the unique applications of virtual reality in many modern contexts, Second Life (SL) offers inimitable opportunities for research and exploration. A review of current research regarding SL has examined the influence of real world social influences in online interactions and what the effects on users may be. This suggests the importance of developing an understanding of the relationship between users' real life and their Second Life, and how the two are related. Some research has begun to reveal the effectiveness of telecommunication and computer simulation with certain clients in the fields of mental health and social work, yet there is a lack of sufficient research done within the context of virtual worlds. The implications for social work intervention in virtual reality could solve persistent concerns with transportation, distance, access to services and education, however questions about the relevance of social work practice in $S L$ and the potential for implementation must be answered.
\end{abstract}

Keywords: Social work, virtual world, Second Life

\section{INTRODUCTION}

Humans as social beings have been inventing ways to improve communication for hundreds of years; from letters to the telephone, from pagers to the cell phone, from email to video conference, and in the last ten years communication technology has taken a giant leap from the internet to computer simulation and virtual reality. The online virtual worlds of Massively Multi-User Role Play Games (MMO-RPGs) are a new wave of interaction and offer to the discerning user a complete immersion in the social experience that transcends distance, language, and even the cultural mores of any given society. Users can plug in anywhere and immediately begin interacting, playing, creating and chatting with other users without regard to their geographic location or even their physical appearance. Time has no meaning in these virtual worlds, and the imagination is the limit as players learn the unique language and culture of this online universe. MMORPGs, or just RPGs as they are known by veteran players, can range from high-resolution action packed gaming, like World of Warcraft, to intricately stylized virtual landscapes where users can gather for social networking, such as Free Realm, There, and Second Life (Wood, 2009).

Imagine being able to enter a multifaceted world with seemingly unlimited opportunities of experience, in which one can create a completely virtual lifestyle, where one's activities are only limited by the creativity of one's inquiry. Here one can learn to easily navigate through an array of experiences often beyond the reach of one's real life means. Second Life is a place where a group can be composed of people who are sitting in their homes located half a world away from each other, where persons who have a

Scott Anstadt, Ph.D., is an Assistant Professor, and Ashley Burnette and Shannon Bradley are MSW Concentration Year Students, all in the Division of Social Work at Florida Gulf Coast University in Fort Myers.

Copyright (C) 2011 Advances in Social Work Vol. 12 No. 2 (Fall 2011), 289-300 
real-life physical limitation can dance all night, where one can appear and disappear in an instant and one's place of origin cannot be traced by anyone around, where people can control who sees and talks to them and where one can even disengage entirely from the virtual world at will. Boellstorf (2008) states "Second Life is a chance to be someone beside yourself, which you can't really do in real life unless you want to lead a double life” (p. 208). This is the beginning of one's Second Life.

Second Life (SL) was created by Linden Labs in 2003 as an online 3D virtual world where people can create "avatars" to interact within the environment and with other avatars (or "avs" as they are frequently known), not only for gaming, but more often for social contact, education, romance, industry, and hundreds of other activities which users can themselves create within the SL metaverse. Second Life is unique in that residents can create and change the virtual landscape by building houses or structures, buying land and adapting their environment to suit their individual needs and desires (McIntosh, 2008). Individuals create avs as virtual representations of their real physical self, and though sometimes these are not accurate representations of their true appearance, the avs serve to navigate the virtual simulators ("SIMS") in place of their human creators. Unlike in real life (RL), SL avatars can transcend the physical boundaries of mere mortals and take the form of humans, animals, computers, mythical creatures, or even as "furries," which are identified as hybrids of any of these forms (Bell, Castranova, \& Wagner, 2009).

Perhaps because there are no real social limits imposed upon the users of SL, it has become a hub of interaction for nearly 15 million users worldwide (Gottschalk, 2010). Of those users, there are hundreds of groups who participate in anything from genderbending avatars, artist guilds, and university students to vampire cults and warring factions of mystical elves. In the presence of the ever-expanding world of virtual reality and computer mediated interaction, it seems as though there is a place for everyone. Even, perhaps, for social workers. However, it is important to remember that, as with all forms of technological advancement, there is a considerable learning curve. In the years since its inception, SL has seen numerous upgrades which have made it easier to use, but new users must still navigate sometimes unfamiliar, and impossibly vast, territory. Many SL groups include tutorials for new users on their SIMS and SL itself has an Island dedicated to an orientation of the virtual world.

Even these thorough classes are sometimes not enough, however, to help "newbies" become completely comfortable using the technology. At the most basic level this means understanding what a "teleport" is and how to use it, how to move between SIMS, join groups, and individualize the basic avatar given upon registering to use SL. As one becomes more immersed in SL, this means learning which groups one wants to join and which groups one would rather avoid, investing dollars for Lindens (SL currency) to purchase "land," and taking classes to begin building one's own Second home, along with other virtually endless activities. This can be an overwhelming process for new users, and sometimes this can even deter users from becoming permanent members of SL. This is one consideration that professionals, including social workers, who are looking to make an entrance into this new medium, must be aware of, for themselves and also for potential clients. 
Social workers often face barriers to service which include distance from clients, time constraints, transportation, and access to services. The virtual world offers a potential solution to many of these barriers. However, the vast expanse of the "metaverse" (internet universe of virtual reality) is still in its infancy, and little research has been done that can conclusively determine the users' expectations of involvement in the virtual world. One of the many questions that remain unanswered is that of the relationship between users' virtual lives and their real lives. For instance, researchers in the field of virtual communications have questioned just how strong the influence of social mores, norms and laws are in internet-based virtual realities like Second Life, and what role accepted institutions such as education play in online interaction and learning (Boelstorff, 2008; Eastwick \& Gardner, 2009; Parti, 2008). Understanding, or at least uncovering, the answers to these and other questions could help to determine the potential for using SL as an educational intervention tool and reveal just how the social work profession can exploit this venue for client-centered interventions.

A review of the literature leads to exploration of relations between the real lives of users and their lives "inworld" (being actively signed in to Second Life), which might suggest the presence of a significant influence of users' real life on the way they conduct their lives inworld. Other questions we suggest concern the associations between what initially brings users to SL, what they do once they are there, and how or why this changes over time. With this information, research in the field of social work can further expand on relevant knowledge about virtual worlds. From there we can begin to explore and predict what role social work practice has in the virtual realm, and whether it could be executed as an effective form of intervention and service delivery. For example, many young adults struggle to come to terms with their sexuality in a society where homosexuality and transgender assimilation is viewed with derision and condemnation. Their real life experiences might prompt them to join SL where using a "genderswapped" avatar is completely appropriate and accepted. In turn, these young adults can interact with other individuals with similar experiences and interests. This begs the question: could the inception of an inworld support group directed by a social worker be beneficial in creating a positive experience for this vulnerable population?

Other groups that meet in SL, and even those individuals who have not yet discovered this online resource, could benefit from similar support systems. This includes individuals without transportation to an agency or facility, individuals with disabilities, or those whose social interaction is limited due to personal illness or caregiver responsibilities. With Second Life, there is unlimited potential for these individuals to open the doors to a huge social network of support and assistance, just by accessing a computer. Using online virtual communication, social workers could reach a larger population of individuals and more diverse groups. And because online meetings can take place at anytime from anywhere, there is a better opportunity for social workers to contact clients and monitor progress. This medium also has the potential to change the face of education for social workers, whether in the classroom or in the field, which could enhance learning and practice skills. 


\section{TECHNOLOGY AND SOCIAL WORK}

The potential for telecommunication as a method for medical, mental health, and social work practice and intervention has been a topic of research since before many of the modern RPG's were launched to the masses. McCarty and Clancy (2002) discuss the potential for "telehealth" technology in social work, asserting that care in mental health was the first telecommunication application used in social work. Internet-based teleconference and videoconference have been established methods of effective communication and treatment for patients of psychiatrists, mental health professionals, and social workers. The authors support the use of online counseling in social work for a number of reasons, including as a solution to barriers of distance and time, as a way of recording client-worker interactions electronically, and even in some cases as a way to enhance personal empowerment of the client, because the physical interaction has been removed, leaving only the verbal, and not face-to-face, communication. However, some disadvantages remain, including confidentiality, informed consent, and liability issues, as well as the lack of non-verbal communication which many practitioners believe to be an integral part of the client-worker relationship (McCarty \& Clancy, 2002). Still, the benefits may outweigh the barriers as redesigning the mental health delivery system could mean better intervention results, agency cost reduction, improved documentation and even distance home visits and employee/intern supervision.

However, while telecommunication has been supported through research as a viable means of intervention and social work service, little has been done to determine whether online virtual reality realms are an equally effective tool. Therefore, it is important to consider other types of technology-based programs that have been used effectively in social work. A recent study by Smokowski and Hartung (2003) analyzed current research on computer simulation games and virtual reality interaction in school social work programs for adolescents and determined that there was a significant improvement in social, behavioral, and problem-solving skills among the participants. They conclude that computer simulation and virtual reality-based interventions, used as a supplement to interpersonal interaction in small groups of adolescents, was an effective tool for school social workers to help the students reach their goals, despite certain barriers, such as access to this technology. Further research in this area is necessary to determine how to implement these programs in schools and develop successful applications for agespecific, population-specific, and skill-specific interventions.

If virtual reality communication interfaces can be implemented and have a significant effect on the outcome of therapy in settings such as school social work, the potential for applications of social work using online metaverses like SL could be great. The challenge in determining the potential effectiveness of this new generation of service is in understanding the various contributing factors and how they might impact the practice of social work in SL. One such factor is the willingness of individuals to participate in online counseling or support groups directed or sponsored by a social worker or mental health professional. Tsan and Day (2007) attempted to determine whether or not variables such as personality and gender can function as predictors of who will seek out or use online counseling in chat rooms with no face-to-face interactions. The findings of the study suggest that females were more likely to accept online services as a means of 
counseling and only the extroverted personality type was accepting of online counseling services, however even the statistical significance of this particular finding was not strong. Most respondents rated face-to-face services above those of online services (Tsan \& Day, 2007). These findings must be considered for future research should social work interventions in virtual worlds become a viable option, though explosive technological advancements will certainly have an effect on clients' willingness to participate in online counseling and group sessions.

Using the Technology Acceptance Model (TAM) to test several hypotheses based on participants' performance expectancy, effort expectancy, and social influence on use of technology in virtual worlds, Fetscherin and Lattemann (2008) found that "the possibility to interact in a 3D environment in combination with Voice over IP plays a pivotal role in user acceptance and technology adoption of virtual worlds” (p. 240). The results reveal that perceived value of communication, or the benefit the users felt could be attained, was more important to participants than perceived usefulness, or how able the users felt they were to learn and use the communication medium. This implies that the visual nature of the avatars as well as the voice technology present in metaverses like Second Life could have a large impact on whether individuals are accepting of online counseling services and find them effective. The findings of the study also suggest that social norms, as well as socio-demographics and the individual's existing attitude toward technology, have an impact on user acceptance of technology (Fetscherin \& Lattemann, 2008). Therefore, the potential influence of these technology characteristics in SL and similar virtual worlds may indeed play a role in determining the presence and usefulness of social work interventions for users in the future.

\section{Real Life and Virtual World Influences}

Much of the body of research emerging on this subject elicits excitement about the potential for SL as a new frontier for social workers to provide services to clients. Consequently, it is imperative that factors such as social influences in virtual worlds and the characteristics and behavior of SL users must be explored. Studies on the emerging world of RPGs and computer-mediated communication have centered on how real life (RL) social influences play a role in SL interactions, behaviors, and even the unique culture, language and etiquette rules present inworld. Eastwick and Gardner (2009) conducted a series of randomized controlled trials in Second Life in order to test an hypothesis that real-world social influence would be present inworld and that participants would display behaviors associated with racial prejudice. In order to test the presence of social influence inworld, researchers used a specific series of behaviors that induce a change in the action of an individual in real life, known as compliance techniques. Racial prejudice was targeted using both an African American avatar and a Caucasian avatar. The data gathered supported their hypotheses, revealing the social influence of the compliance techniques had an effect on the avatar-participants (presumed to be their human creators) and that they were more likely to respond with compliance to the Caucasian avatar than to the African American avatar. These findings suggest the importance of gathering exploratory data on users' RL and SL interactions so that any associations may be identified for use in further studies. 
With the strong likelihood that real social influences are present within the virtual world of SL, social workers can begin to explore how RL influences the creation of the SL experience and how this may be related to the real lives of SL users. However, there is some evidence that other social influences are absent within the virtual walls of Second Life and RPGs like it, which could have an impact on the implementation and effectiveness of social work interventions executed inworld. Parti (2008) explores how the relative freedom of expression and creation inworld can both enhance and degrade real-world social mores. The author discusses the implications of social construction in virtual reality and how this relates to the legal system in the real physical world. Bell et al. (2009) comment that there is a complete lack of government jurisdiction over virtual worlds as they, like the internet, are not a part of any country on Earth and, therefore, do not fall under the typical auspices of the law.

Parti (2008) questions the integrity of the individual's real personality as she/he continues to become immersed in virtual reality, even perhaps eroding without the user's knowledge, as she/he places more attention, time, and energy into SL communications. Because virtual reality is a replica of reality, and one highly guided by imagination and illusion, it is difficult to know how strongly traditional social moral-ethical norms apply inworld. In her meta-analysis of several qualitative case studies done on users of RPGs and MUDs (Role Play Games and Multi-User Domains/Dungeons), Parti (2008) illuminates several examples of how the user dissociates from her/his avatar and considers the avatar to have a different personality, one that is enhanced, perfected, and capable of far more than the user her/himself. In doing so, belief in one's own ability to perform these activities and interpersonal interactions deteriorates, and she/he experiences a de-empowerment of self. Because the user does not identify with the avatar self, she/he does not necessarily feel satisfaction or a sense of success when the avatar succeeds, and she/he does not carry acquired social skills into RL interactions (Parti, 2008). Additional concerns include social learning and community mentality which can affect a moral attitude change and encourage negative deviant behavior such as computer hacking. This affects the population of children and adolescents most significantly, and teaches negative skills and behaviors that could carry into their real life future.

This information carries serious implications for social work practice in RPGs such as Second Life. The role of the social worker in SL will need to be diverse and flexible, with social workers acting as counselors as well as advocates, and adapting guidelines for confidentiality and legality without detracting from ethical service. Knowing the social and psychological barriers to service can help social workers to anticipate, and be prepared to address, the needs of their clients. Furthermore, understanding who is most likely to use SL and in what capacities they use it will help to define the methods through which these individuals can be reached and helped.

\section{Real Life and Second Life Relationships}

But what, if any, relationships exist between the individual in real life and the avatar in Second Life? Whether or not any associations can be made between the actions of the one and the same user-avatar can be determined with further research in this area. With this knowledge as a foundation, further predictions based on the user-avatar relationship 
can be made and implications for social work interventions explored. In a related study, Belisle and Bodur (2010) attempted to determine if personality types of SL users could be perceived by observing their avatar. They conducted a study in which 103 SL users sent in a picture of their avatar and filled out a questionnaire about their participation, personality traits and socio-demographic information. Then, seven separate participants were asked to predict personality characteristics of the real person by looking at their avatar. These seven participants were selected on the basis of their high level of experience with SL and also filled out an online questionnaire. The findings of the study suggest that certain physical characteristics of avatars serve as cues to form impressions of the real personality of their creator, and that experienced users can form accurate impressions (Belisle \& Bodur, 2010).

The study also revealed an inverse relationship between some avatar characteristics and the demographic information of the creator. Taking this information into account, it is conceivable that social workers could attempt counseling and group work with avatars as representations of clients. However, they would need to develop a high level of skill and experience with Second Life and develop an understanding of the real life circumstances of SL users.

Stalker (2007) predicted that in the real world social role diversity is determined by several factors that can affect the number of social roles of an individual, the strength of her/his social network, her/his adaptability to life circumstances, and her/his overall level of well-being. Additionally, characteristics affect an individual's opportunities as well as create barriers to reaching fulfilling interpersonal engagement. These barriers include social context, patterns of employment, and the amount of time spent engaging in pleasurable activities. Through time-diary collection of data, Stalker (2007) determined that role diversity was positively affected by marital status, the presence of children, employment, and gender. Subsequently, the level of interpersonal engagement and the strength of the social support network of the individual were positively affected by these factors. Ultimately, a less diverse social role resulted in a smaller social network, less time spent interacting and communicating with others, and weakened resiliency to varying life circumstances.

In light of these findings, the consideration of social work practice within virtual reality must include an understanding of the relationship of the RL user to her/his SL avatar. Research focusing on the relationship between the user's ability to manipulate the physical appearance of her/his avatar, or change demographic information, and the level of effective communication attained between client and social worker could begin to uncover ways in which to equip the social worker to make assumptions about the user/client. In coming to an understanding about the potential motivations that bring users to SL, and how this could relate to the user's real life, social workers can begin to formulate treatment options. For instance, an individual without a high degree of role diversity might be motivated to join SL to seek out interactions and a social network, and the reasons for limited role diversity could be an ongoing illness in the family or disability. This supports the need for a social work intervention that could potentially take place within Second Life itself. Further research in the form of qualitative data analysis could examine the existence of a relationship similar to the one in this situation and 
provide an insight into the role of social work interventions in virtual reality-based communication.

The impact of certain demographic factors on interactions within virtual worlds was explored by Isabella (2007) in a study conducted to determine how ethnographic differences between players of MUDs (“multiple user domains") might change their interactions and communication. The study was based on the assumption that online communities mirror to some extent their real-world counterparts. Through covert and participant observation in two MUDs that were relatively isolated to two precise regions, one in Italy and the other in Canada, Isabella (2007) first recorded group interactions before engaging players, and then proceeded to join a group of players and record interactions from within the group. Her results indicated that there were certain differences in the communication and community engagement styles of each region. In the Italian MUD, with little effort, the researcher was able to observe covertly before engaging in the groups. After disclosing her identity and real motivations, the players did not seem to change their impressions of, or interactions with, her. However, in the Canadian MUD, the researcher had difficulty joining a group after her initial observations, and it was only when she disclosed her purpose to the leader of a group, and the leader informed all of the players that she was a researcher and needed their participation, that she was able to collect any data.

These results indicate that the physical contexts of the users may have an influence on their attitude and the games that they play. Evidence supports that there are indeed differences in the culture and communication of users from different demographic backgrounds, although the full extent of these influences has not been explored. Additionally, the social context of the virtual world itself must be considered as its own entity and as a unique culture separate from that of the real-world culture.

In gathering data on Second Life through participant observation, Gottschalk (2010) discovered both a blurring of and a separation between the real lives of the users/avatars he interviewed and their life on SL. Gottschalk (2010) also illuminates that while users can have many avatars that look nothing like them, they communicate in essentially the same ways with all of their avatars. Based on the information gathered, Gottschalk (2010) focuses on further suggestions for research on particular aspects of Second Life. These include the progression of the "newborn" avatar and the changes they make as they develop over time, as well as the vast potential for educating students inworld. Finally, Gottschalk (2010) suggests that factors such as gender, age, race, residence and physical appearance do not matter in Second Life as much as they matter in real life, and postulates that the real information worth gathering is "how they enact their identity, what their motivations are for participating in Second Life, what they typically do there, what they discover about themselves, and how they negotiate the boundaries between real life and Second Life" (Gottschalk, 2010, p. 520). These themes will be discussed in light of their impact to research in the field of social work and social work practice.

One of the drawbacks of the previous research on virtual worlds referred to above is that these data were collected outside of the virtual world instead of while users were still immersed in it. Future research taking place within the context of SL could bridge this 
gap in research design. Currently, most data collection methods employ the use of listservs that invite virtual world users to access a database or website outside of the context of Second Life in order to provide survey or other quantitative data. However, by collecting data from participants while they are still inworld, the integrity of the unique culture of Second Life is simultaneously maintained while the data is being gathered, providing the possibility that answers to research questions will be more accurate and representative. These include questions about what motivates people to join Second Life, what activities and interactions they participate in inworld, what the context of their real lives is, and whether or not an association exists between the two. Additionally, further examination of research results would relate these findings specifically to the field of social work in the future.

In a study which illustrates data collection within the context of virtual worlds, Bell and colleagues (2009) used a method of collecting data within Second Life called Virtual Assisted Self Interview (VASI) with a tool known as Virtual Data Collection Interface (VDCI). The study collected results of a survey measuring demographics and quality of life of users given on a large scale to users all over Second Life while they were present inworld. What researchers discovered suggests that Second Life users are quite possibly unique from users of other virtual worlds and establishes that the VDCI technology is an effective tool for gathering representative information from SL users inworld (Bell et al., 2009). There is an assumption that gathering data while users are still present in Second Life not only inspires users to take and complete a survey, but also preserves the environment and culture that users are being surveyed about. This is presumed to increase the likelihood that answers will reflect participants' mentality and perspective as a user of Second Life. Therefore, further research is needed to explore similar methods of data collection.

Based on the social constructionist perspective, in which individuals base their understanding of, and participation in, their reality upon socially constructed views, the presence of a significant association between the participants' real lives and their Second Life experience could play an important role in determining future research in this area. We suggest that among the variables being measured are included the users' participation in groups in RL and SL, their attitudes toward meeting their SL friends in RL, their demographics and activities both in RL and SL, their relationships in RL and SL, their motivations in RL and SL, the frequency of participation in SL, and their RL social interactions. By examining the associations between these variables, predictions can be made which allude to important characteristics of users and their experiences and lead to insights into the potential implementation and efficacy of social work interventions in the virtual world of Second Life.

\section{DISCUSSION}

Current research has developed a substantial base of understanding about many aspects of online virtual world use, including the cultural differences between Second Life and real life as well as the use of this technology for purposes such as marketing, anthropological research, and educational uses in online interactions (Bell et al., 2009; Eastwick \& Gardner, 2009; McCarty \& Clancy, 2002; Smokowski \& Hartung, 2003). 
However, there is a gap in the current research regarding what motivates users to join in the online melee and how the real world and the online world interact with each other. This is mainly in regards to interpersonal communication and how individuals function (Gottschalk, 2010). Current research methods used inworld are improving the quality of the data collected and future advances in data collection can provide a more accurate representation of information. Research in the field of social work is uniquely designed to examine how individuals and groups interact socially, and can be adapted to aid in the exploration of these interactions in Second Life and other similar online social venues. In order to advance and bridge barriers to social work practice through the utilization of modern technology, there must first be an understanding of the nature of online worlds such as Second Life and their potential as a tool for social work interventions in the future.

\section{Implications for Research}

Some limitations exist in data collection in online virtual worlds, including concerns about maintaining participant confidentiality in a venue where computer hacking is somewhat prevalent (Bell et al., 2009; McCarty \& Clancy, 2002). Also, research in the field of social work must adhere to the code of ethics and remain cognizant of the unique context of online virtual reality use. Additional considerations should be made toward users with developmental and physical disabilities.

Further limitations continue to persist due to the current state of technological advancements and constraints. Computer-based research can only extend so far as modern technology allows it to, and there is a lack of established efficacy of online data collection within Second Life itself. This is evident in interactions with both SL users as well as the educational communities present within SL, especially in regards to the unique cultural aspects present inworld.

Additional considerations must also be made for the future of research protocols, and the policies and ethical requirements of the Internal Review Board currently do not take into account the quickly advancing technology of online virtual worlds. Given the unique technical potential of SL, this puts the responsibility on the researcher to take pains to be as ethical as possible when conducting research. Avatars may not be humans, but the person controlling, and more importantly experiencing, the avatar most certainly is, and therefore is susceptible to all of the vulnerabilities of more typical research subjects. The future of SW research also means a new future for IRB research protocols.

Should inworld data collection be successful, this opens the doors for further and more pertinent research which is capable of maintaining the integrity of the unique sociocultural atmosphere of Second Life and similar virtual worlds. This may become a key strength of the research in this area.

\section{Implications for Social Work Practice}

Further research in the field of social work on the use of online virtual worlds like Second Life could illuminate the future of social work practice and interventions. Social workers may be able to use this tool to advance and broaden their scope of practice, 
bringing technology and social work together and redefining how we reach individuals and groups through interactions, counseling, and advocacy. For instance, individuals with disabilities or their homebound caregivers could benefit exponentially from this service, connecting them to social interactions and social support networks without taxing them physically (Heron, Gentle, personal communication, April 29, 2011). This could reduce the impact of their disability or the disability of a loved one on their overall well-being.

Special consideration must be made for ethical demands of practice, including issues of safety, prevention, and mandatory reporting. Questions, such as what a social worker is responsible for if a client or group member expresses suicidal ideation while communicating via Second Life interactions, must be given the utmost consideration before beginning this potential venture. These questions are likely to produce opinions and concerns across the spectrum of professional social workers and will need to be defined ethically as well as legally.

However, other questions still remain unanswered and may remain so for some time into the future. One major question that these researchers ask is: Will online counseling and the use of upcoming technological advancements ultimately enhance real life interpersonal interactions and the development and use of social support networks and social skills in real life in the future? Are social workers now participating in a medium that will eventually encompass and promote empowering clients to access their real life resources in the real world? Only time will tell.

\section{References}

Belisle, J. F., \& Bodur, H. O. (2010). Avatars as information: Perception of consumers based on their avatars in virtual worlds. Psychology and Marketing, 27(8), 741-765. doi: 10.1002/mar.20354

Bell, M. W., Castranova, E., \& Wagner, G. G. (2009). Surveying the virtual world: A large scale survey in Second Life using the Virtual Data Collection Interface (VCMI). Data Documentation, 44, 1-61.

Boellstorff, T. (2008). Coming of age in Second Life: An anthropologist explores the virtual human. Princeton: Princeton University Press.

Eastwick, P. W., \& Gardner, W. L. (2009). Is it a game? Evidence for social influence in the virtual world. Social Influence, 4(1), 18-32. doi: 10.1080/15534510802254087

Fetscherin, M., \& Lattemann, C. (2008). User Acceptance of virtual worlds. Journal of Electronic Commerce Research, 9(3), 231-242.

Gottschalk, S. (2010). The presentation of avatars in second life: self and interaction in social virtual spaces. Symbolic Interaction, 33(4), 501-525. doi:

10.1525/si.2010.33.4.501

Isabella, S. (2007). Ethnography of online role-playing games: the role of virtual and real contest in the construction of the field. Forum: Qualitative Social Research, 8(3), 116. 
McCarty, D., \& Clancy, C. (2002). Telehealth: Implications for social work practice. Social Work, 47(2), 153-161.

McIntosh, K. (2008). The social construction of virtual space. Michigan Sociological Review, 22(1), 196-214.

Parti, K. (2008). Deviances in the virtual reality or the character-altering power of virtual communities. European Journal of Crime, Criminal Law \& Criminal Justice, 16(3), 325-343. doi:10.1163/157181708X333373

Smokowski, P. R., \& Hartung, K. (2003). Computer simulation and virtual reality: enhancing the practice of school social work. Journal of Technology in Human Services, 21(1/2), 5-30.

Stalker, G.J. (2007). Measuring diversity in daily social contact: The contribution of social context, work and leisure on the opportunity for engagement. Springer Science+Buisness Media, 86, 275-295. doi: 10.1007/s11205-007-9115-5

Tsan, J. Y., \& Day, S. X. (2007). Personality and gender as predictors of online counseling use. Journal of Technology in Human Services, 25(3), 39-55.

Wood, D. (2009). Real life access to "Second Life" worlds: The potential, the problems and the possibilities for a barrier-free future. The International Journal of Diversity in Organizations, Communities, and Nations, 8(6), 139-148.

\section{Author note:}

Address correspondence to: Scott Anstadt, Ph.D., Division of Social Work, Florida Gulf Coast University, 10501 FGCU Blvd., South, Fort Myers, FL 33965-6565. Email: sanstadt@fgcu.edu 